\title{
Diagnostic accuracy assessment of molecular prediction model for the risk of NAFLD based on MRI-PDFF diagnosed Chinese Han population
}

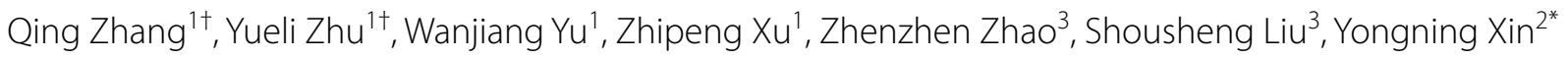
and Kuirong LV ${ }^{1 *}$

\begin{abstract}
Background: Several molecular prediction models based on the clinical parameters had been constructed to predict and diagnosis the risk of NAFLD, but the accuracy of these molecular prediction models remains need to be verified based on the most accurate NAFLD diagnostic method. The aim of this study was to verify the accuracy of three molecular prediction models Fatty liver index (FLI), NAFLD liver fat score system (NAFLD LFS), and Liver fat (\%) in the prediction and diagnosis of NAFLD in MRI-PDFF diagnosed Chinese Han population.

Patients and methods: MRI-PDFF was used to diagnose the hepatic steatosis of all the subjects. Information such as name, age, lifestyle, and major medical histories were collected and the clinical parameters were measured by the standard clinical laboratory techniques. The cut-off values of each model for the risk of NAFLD were calculated based on the MRI-PDFF results. All data were analyzed using the statistical analysis software SPSS 23.0.

Results: A total of 169 subjects were recruited with the matched sex and age. The ROC curves of FLI, NAFLD LFS, and Liver fat (\%) models were plotted based on the results of MRI-PDFF. We founded that the accuracy of FLI, NAFLD LFS, and Liver fat (\%) models for the prediction and diagnosis of NAFLD were comparative available in Chinese Han population as well as the validity of them in other ethnics and regions.

Conclusions: The molecular prediction models FLI, NAFLD LFS, and Liver fat (\%) were comparative available for the prediction and diagnosis of NAFLD in Chinese Han population. MRI-PDFF could be used as the golden standard to develop the new molecular prediction models for the prediction and diagnosis of NAFLD.
\end{abstract}

Keywords: Non-alcoholic fatty liver disease, MRI-PDFF, Molecular prediction model

*Correspondence: xinyongning@163.com; Ivkr5169@163.com

${ }^{\dagger}$ Qing Zhang and Yueli Zhu authors contributed equally to this work

1 Department of Radiology, Qingdao Municipal Hospital, 5 Donghaizhong

Road, Qingdao 266071, Shandong Province, China

2 Department of Infectious Disease, Qingdao Municipal Hospital, 1

Jiaozhou Road, Qingdao 266011, Shandong Province, China

Full list of author information is available at the end of the article

\begin{abstract}
Introduction
Non-alcoholic fatty liver disease (NAFLD) has become the most prevalent chronic liver disease over the past several years, and the average prevalence of NAFLD approximately $25 \%$ in the world, and even over the $30 \%$ in some districts [1-3]. The spectrum of NAFLD ranges from non-alcoholic fatty liver (NAFL), non-alcoholic steatohepatitis (NASH), fibrosis, cirrhosis, and even the hepatocellular carcinoma [4, 5]. Accurate predicating and diagnosing the risk of NAFLD and taking actions to
\end{abstract}

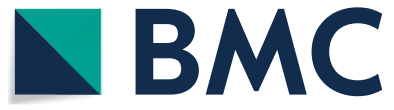

(c) The Author(s) 2021. Open Access This article is licensed under a Creative Commons Attribution 4.0 International License, which permits use, sharing, adaptation, distribution and reproduction in any medium or format, as long as you give appropriate credit to the original author(s) and the source, provide a link to the Creative Commons licence, and indicate if changes were made. The images or other third party material in this article are included in the article's Creative Commons licence, unless indicated otherwise in a credit line to the material. If material is not included in the article's Creative Commons licence and your intended use is not permitted by statutory regulation or exceeds the permitted use, you will need to obtain permission directly from the copyright holder. To view a copy of this licence, visit http://creativecommons.org/licenses/by/4.0/. The Creative Commons Public Domain Dedication waiver (http://creativeco mmons.org/publicdomain/zero/1.0/) applies to the data made available in this article, unless otherwise stated in a credit line to the data. 
prevent the progression of NAFLD shows great significance. Liver biopsy remains is the diagnostic gold standard of NAFLD, but the clinical application of liver biopsy is not extensive acceptable due to some defects such as invasiveness, sample error, and operator-dependent $[6$, 7]. Some noninvasive imaging diagnosis methods such as ultrasonography, computed tomography and controlled attenuation parameter had been conventional used in clinical diagnosis of NAFLD, but the accuracy could match to biopsy and need to be improved [8-10].

Magnetic resonance imaging derived proton density fat fraction (MRI-PDFF) is a newly developed and abundant applied NAFLD diagnose method recently years which can assess the liver fat over the entire liver accurately and MRI-PDFF possesses the higher repeatability and reproducibility $[8,11-13]$. Imajo et al. reported that MRIPDFF could accurate classify the grades of steatosis in biopsy-proven NAFLD patients [14]. Subsequently, Middleton et al. reported that MRI-PDFF has the high accuracy to both classify and predict histological steaotsis grade and change in histological steatosis grade in children with NAFLD [15]. A meta-analysis conducted by $\mathrm{Gu}$ et al. suggested that MRI-PDFF has excellent diagnostic value for assessment of hepatic fat content and classification of histologic steatosis in NAFLD patients [16].

In addition to the biopsy and imaging diagnostic methods, some molecular prediction models had been constructed and used for the prediction of development risk of NAFLD and the hepatic fat content based on the clinical parameters. As early as 2006, Bedogni et al. built a hepatic steatosis predictor which named "Fatty liver index (FLI)" using the clinical parameters such as gender, age, ethanol intake, gammaglutamyl-transferase (GGT), body mass index (BMI) and so on [17]. In 2009, Kotronen et al. reported a NAFLD liver fat score system (NAFLD LFS) which could predict the fat score in liver according to the metabolic syndrome (MS), type 2 diabetes (T2D), fasting insulin, AST and ALT. In addition, Kotronen et al. developed a model to predict the liver fat percent based on the above factors [18]. Subsequently, many studies had diagnostic the risk of NAFLD and the fat content in liver according to the molecular prediction model [19-22], but the specificity and sensibility were remains need improved.

In fact, PDFF testing has not yet been used in medical activities due to the lack of machines and relevant clinical data. For the NAFLD patients who can't be diagnosed by MRI-PDFF, the molecular prediction models could be regarded as the preferred tools to predict the development risk of NALFD. In this study, all the NAFLD patients and health controls that we recruited were subjected to the MRI-PDFF, and the FLI, NAFLD LFS, and Liver fat (\%) models were used to calculate the liver fat score and liver fat percent in all the subjects. The diagnostic accuracy of FLI, NAFLD LFS, and Liver fat (\%) models were investigated according to the results of MRI-PDFF.

\section{Patients and methods \\ Participants}

From March 2019 to August 2019, a total of 169 participants were recruited randomly from the Department of Gastroenterology and healthy examination center of Qingdao Municipal Hospital that including NAFLD patients who diagnosed by ultrasonography and health peoples. This study was conducted in strict compliance with the Declaration of Helsinki [23]. This study was approved by the Ethics Committee of Qingdao municipal hospital and the written informed consents were obtained from all Participants. All the subjects were pre-diagnosis by B-type ultrasonography. All the subjects were Chinese Han population, and the age and sex were matched. Subjects with the following cases were excluded: (1) excess alcohol consumption (males $>210 \mathrm{~g} / \mathrm{w}$, females $>140 \mathrm{~g} / \mathrm{w}$ ) or cigarettes; (2) accompanied with viral hepatitis, drug-induced hepatitis, autoimmune hepatitis, or other factors induced chronic liver diseases; (3) suffering from tumors or surgery in nearly 2 years.

\section{Clinical parameters collection}

The basic information of each participant such as name, age, lifestyle, and major medical histories were collected by a standard questionnaire. Subjects with excess alcohol intake and other liver-related diseases were excluded from this study. The height, weight, waist circumference (WC) and hip circumference (HC) of each participant were measured by two professional inspectors. The body mass index (BMI) was calculated based on the equation as weight $(\mathrm{kg}) / \mathrm{height}^{2}\left(\mathrm{~m}^{2}\right)$. The diastolic blood pressure and systolic pressure of each participant were measured twice with a $5 \mathrm{~min}$ interval and the average values were recorded. Each participant was subjected to an overnight fasting and the venous blood was sampled. The serum levels of aspartate aminotransferase (AST), alanine aminotransferase (ALT), gamma-glutamyltranspeptidase (GGT), triglyceride (TG), total cholesterol (TC), lowdensity lipoprotein (LDL), high-density lipoprotein (HDL) and fasting serum insulin were measured by the standard clinical laboratory techniques, respectively. The values of FLI, NAFLD LFS, and Liver fat (\%) were calculated according to the formula in the Table 1.

\section{MRI-PDFF examination}

Each participant was subjected to the MRI-PDFF examination. Participants were scanned using a 3.0 T MRI 
Table 1 The calculation formula for FLI, NAFLD LFS and liver fat (\%)

\begin{tabular}{|c|c|c|}
\hline Score & Algorithm & References \\
\hline FLI & $\begin{array}{l}\mathrm{FLI}=\left(e^{\left.0.953^{*} \log \text { (triglycerides }\right)+0.139^{*} \mathrm{BMI}+0.718^{*} \log } e^{(G G T)+0.053^{*} \text { waist circumference }-15.745}\right) /\left(1+e^{0.953^{*} \log } e^{\text {(triglycerides })+0.139^{*} \mathrm{BMI}+0.718^{*} \log } e^{(G G T)+}\right. \\
\left.0.053^{*} \text { waist circumference }-15.745\right) * 100\end{array}$ & [17] \\
\hline NAFLD LFS & $\begin{array}{l}\text { NAFLD LFS }=-2.89+1.18 * \text { metabolic syndrome }(\text { yes }=1 / \text { no }=0)+0.45 * \text { type } 2 \text { diabetes }(\text { yes }=2 / \text { no }=0)+0.15 * \text { fasting } \\
\text { serum insulin }(\mathrm{mU} / \mathrm{L})+0.04 * \mathrm{AST}(\mathrm{U} / \mathrm{L})-0.94 * \mathrm{AST} / \mathrm{ALT}\end{array}$ & {$[18]$} \\
\hline Liver fat (\%) & $\begin{array}{l}\left.10^{(-0.805+0.282 *} \text { metabolic syndrome (yes }=1 / \mathrm{no}=0\right)+0.078 * \operatorname{type} 2 \text { diabetes }(\text { yes }=2 / \mathrm{no}=0)+0.525 * \lg (\mathrm{fs}-\operatorname{insulin}[\mathrm{mU} / \mathrm{L}])+0.521 * \lg (\mathrm{fs}-\mathrm{AST}[\mathrm{U} / \mathrm{L}])-0.454 * \\
\lg (\text { AST/ALT }))\end{array}$ & [18] \\
\hline
\end{tabular}

BMI, body mass index; FLI, fatty liver index; GGT, gamma-glutamyltransferase; NAFLD, non-alcoholic fatty liver disease; NAFLD LFS, non-alcoholic fatty liver disease liver fat score; AST, aspartate aminotransferase; ALT, alanine aminotransferase

Ingenia system (Philips Healthcare, Best, the Netherlands) with a six-echo mDixon-quant gradient-echo sequence, the filp angle was $3^{\circ}$ and the time of repetition (TR) was $5 \mathrm{~ms}$ [24]. MR imaging analysis was performed by two trained image analysts who were blinded to this project. MR images were reviewed by the Osirix software and manually placed circular regions of interest (ROIs) in each of the nine Couinaud liver segments of the MR imaging PDFF maps in each participant. PDFF in each of the nine ROIs was recorded and the entire liver PDFF values were calculated as the mean of the nine PDFF values. Participants with a MRI-PDFF value $\geq 6.4 \%$ was diagnosed as the NAFLD patients [25].

\section{Diagnostic criteria of MS and T2D}

Metabolic syndrome was diagnosed according to the diagnostic criteria of Chinese Diabetes Society, and three or more of the following items are sufficient for diagnosis: (1) $W C \geq 90 \mathrm{~cm}$ (male) and $85 \mathrm{~cm}$ (female); (2) $\mathrm{FPG} \geq 6.1 \mathrm{mmol} / \mathrm{L}$ or 2 -h PG levels $\geq 7.8 \mathrm{mmol} / \mathrm{L}$ after a $75 \mathrm{~g}$ oral glucose-tolerance test or have been diagnosed with diabetes; (3) blood pressure $\geq 130 / 85 \mathrm{mmHg}$ or had been diagnosed with hypertension; (4) $\mathrm{TG} \geq 1.7 \mathrm{mmol} / \mathrm{L} ; 5$ ) $\mathrm{HDL}<1.04 \mathrm{mmol} / \mathrm{L}$ [26]. T2D was defined as typical symptoms of diabetes (polydipsia, polyuria, polyphagia, and weight loss) plus random plasma glucose $\geq 11.1 \mathrm{mmol} / \mathrm{L}, \quad$ or $\quad \mathrm{FPG} \geq 7.0 \mathrm{mmol} / \mathrm{L}$, or OGTT 2 h PG $\geq 11.1 \mathrm{mmol} / \mathrm{L}$ [26].

\section{Statistical analysis}

All data were analyzed using the statistical analysis software SPSS 23.0. All the volunteers were categorized into the NAFLD group and health group according to the clinical characteristics and MRI-PDFF test. Qualitative data composition ratios were compared using chisquare analysis, and ages between NAFLD group and health group were compared using student $t$-test. The paired-samples t-test was used to assess the difference in predicted liver fat (\%) and actual liver fat (\%) detected by MRI-PDFF, while Spearman's rank correlation test was used to assess the correlations of them. We used the receiver operating characteristic (ROC) analysis to redetermine the cut-off values of FLI, LFS, and liver fat (\%) in diagnosing NAFLD. The sensitivity and specificity of cutoff value were calculated. "Youden index" was used to determine the optimal cutoff, Sensitivity-(1-specificity), and the maximum value of the index value is the optimal cutoff value. $P<0.05$ was considered statistically significant.

\section{Results}

Clinical characteristics of included participants

According to clinical characteristics and MRI-PDFF results, participants were divided into two groups, NAFLD group $(n=109)$ and health group $(n=60)$. Characteristics and disease composition of NAFLD

Table 2 Characteristics and disease composition of NAFLD and health groups

\begin{tabular}{lllrr}
\hline & NAFLD $(\mathbf{n = 1 0 9 )}$ & Health group $(\mathbf{n}=\mathbf{6 0})$ & $\mathbf{t} / \mathbf{X}^{\mathbf{2}}$ & $\boldsymbol{P}$ value \\
\hline Age $($ mean \pm SD), years & $42.2 \pm 12.8$ & $42.0 \pm 12.8$ & 0.119 & 0.906 \\
Male, $\mathrm{n}(\%)$ & $70(64.22)$ & $26(43.33)$ & 6.880 & 0.009 \\
BMI (mean \pm SD), $\mathrm{kg} / \mathrm{m}^{2}$ & $28.3 \pm 4.1$ & $25.1 \pm 4.0$ & 0.478 & $<0.001$ \\
T2D, $\mathrm{n}(\%)$ & $12(11.01)$ & $2(3.33)$ & - & 0.142 \\
MS, n (\%) & $46(42.20)$ & $11(18.33)$ & 9.863 & 0.002 \\
\hline
\end{tabular}

BMI body mass index, NAFLD non-alcoholic fatty liver disease, T2D type 2 diabetes, MS metabolic syndrome

Data were compared by t-test, fisher-test or chi-square analysis when appropriate. $P<0.05$ was considered statistically significant 
group and health group were shown in Table 2. There was no significant difference in age between the two groups $(P>0.05)$. The proportion of male patients with NAFLD was higher than male of the control group $(P=0.009)$. BMI value was significant higher in the NAFLD group $(28.3 \pm 4.1)$ than in the health group $(25.1 \pm 4.0)(P<0.001)$. The proportion of patients with metabolic syndrome in NAFLD group $(42.20 \%)$ was significantly higher than that in health group (18.33\%) $(P=0.002)$. There was no significant difference in the composition of diabetic patients between the two groups $(P>0.05)$.

\section{NAFLD and fatty liver index (FLI)}

In order to investigate the accuracy of FLI on the diagnosis of NAFLD, the ROC curve was plotted based on FLI score and MRI-PDFF diagnosis results (Fig. 1). Youden index was used to calculate the optimal cutoff value (cut-off $\geq 37.643$ ), with a sensitivity of $87.0 \%$ and specificity of $58.5 \%$. The area under the ROC curve (AUROC) was 0.78 (95\% CI: 0.71-0.86). In the original literature [17], hepatic steatosis could be diagnosed when the FLI score $\geq 60$. In addition, in our ROC curve, the FLI score 60 possesses a sensitivity of $59.8 \%$ and specificity of $81.1 \%$. These results suggested that this molecular prediction model was comparative superior.

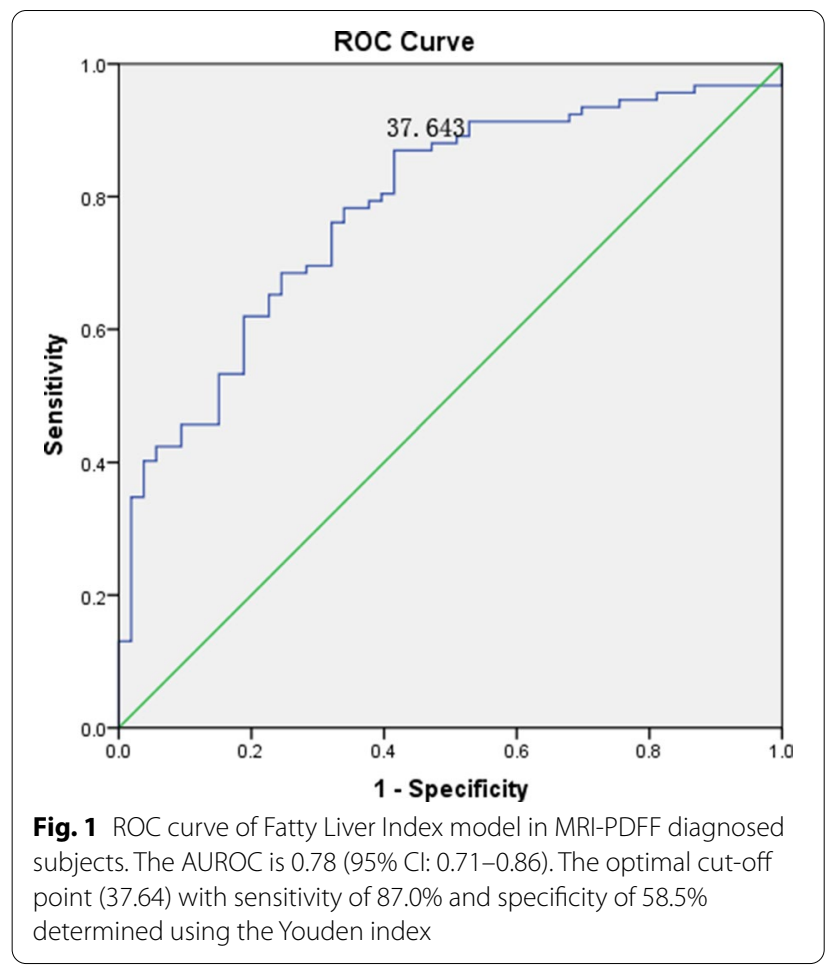

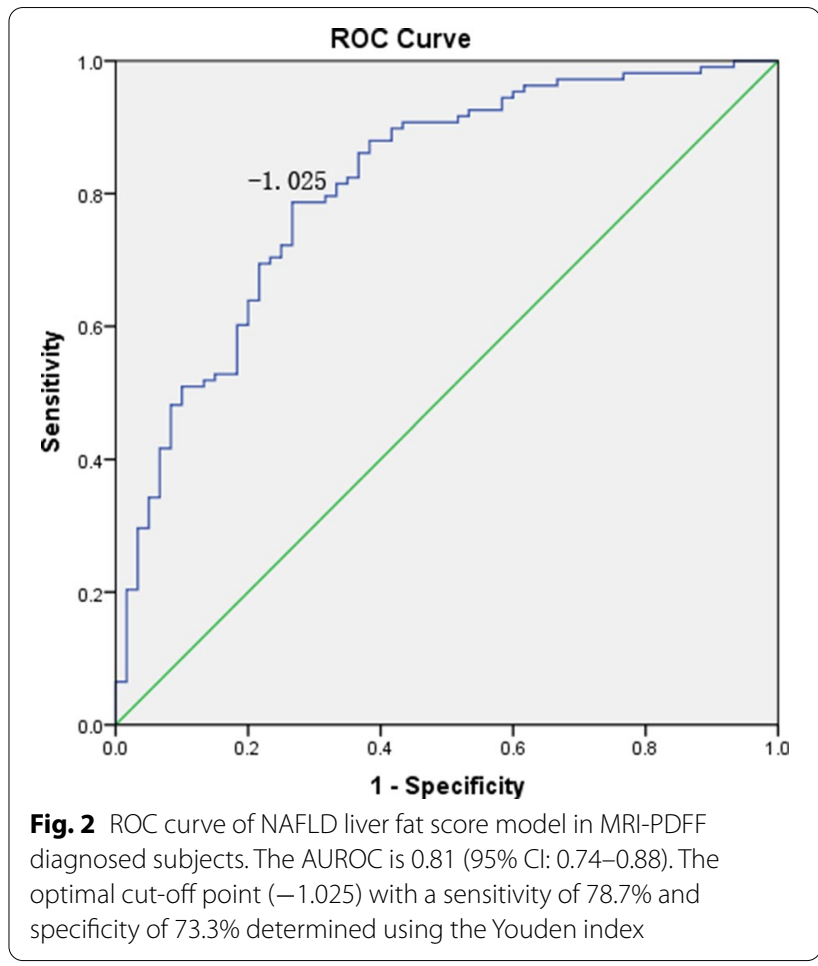

\section{NAFLD and NAFLD liver fat score (NAFLD LFS)}

In order to investigate the accuracy of NAFLD LFS on the diagnosis of NAFLD, we plotted ROC curve based on NAFLD LFS and MRI-PDFF diagnosis results (Fig. 2). Youden index was applied to determine the optimal cutoff value for NAFLD, and we found that the NAFLD LFS values greater than -1.025 could predict the development of NAFLD (sensitivity $=78.7 \%$, specificity $=73.3 \%$ ). The AUROC was 0.81 (95\% CI: 0.74-0.88). The optimal cutoff value in the original literature (LFS $\geq-0.640)$ [18] was substituted into this ROC curve and the corresponding sensitivity was $67.6 \%$ and specificity was $78.3 \%$. When sensitivity (86\%) was the same as that of the original text, the cutoff value was -1.4342 , which was lower than the optimal cutoff value. These results suggested that this molecular prediction model was comparative superior.

\section{NAFLD and liver fat (\%)}

Kotronen $\mathrm{A}$ et al. developed a formula to predict the liver fat percentage. In this study, the paired-samples $\mathrm{t}$-test was used to assess the difference in predicted liver fat percentage and actual liver fat percentage which was detected by MRI-PDFF, and the significant difference of the liver fat between the two results was observed $(t=10.357, P<0.001)$. Spearman's rank correlation test was used to assess the correlation of them, as the results shown, the correlation coefficient was $0.649(P<0.001)$, 


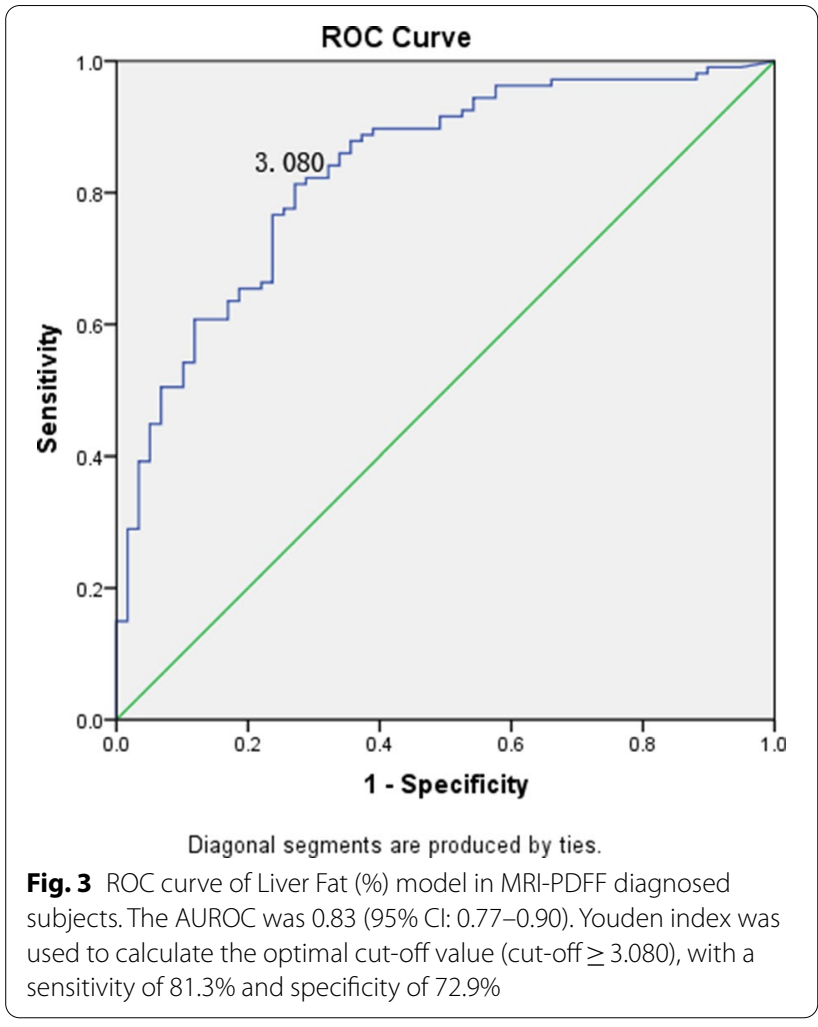

which indicated that the predicted value of liver fat by the formula is moderately correlated with the actual value. We plotted ROC curve based on predicted liver fat percentage and NAFLD diagnosis results (Fig. 3), and the AUROC was 0.83 (95\% CI: 0.77-0.90). Youden index was used to calculate the optimal cut-off value (cutoff $\geq 3.080$ ), with a sensitivity of $81.3 \%$ and specificity of $72.9 \%$.

\section{Discussion}

NAFLD is defined as the excess accumulation of fat in the liver and tightly associated with the obesity, T2D, MS, insulin, and dyslipidemia [18, 27]. Hepatic steatosis is the early stage of NAFLD and could progress to the NASH [28]. Accurate prediction and diagnosis of NAFLD in the early stage will take good effects on the prevention of NAFLD progression and the development of various complications. In recent years, MRI-PDFF had been used to diagnose the hepatic steatosis in several countries and the reliable accuracy, sensitivity and specificity had been verified $[14,15,25,29]$. Besides the imaging and pathological diagnosis, some molecular prediction models based on the clinical parameters, genetics information, and serum biochemical factors had been constructed for the prediction of the risk of NAFLD frequently [30-32]. Chinese people's diet structure, obesity type and other susceptible factors were not exactly the same as those of other regions, therefore the sensitivity and specificity of the models were remain worth discussed based on the most accurate NAFLD diagnostic method such as MRIPDFF in different ethnics and regions. For suspected NAFLD, biopsy was recommended for confirmation. Since biopsies were invasive, we need a reliable, safe diagnostic alternative to biopsy. In this study, we performed the MRI-PDFF examination as the NAFLD diagnostic gold standard to verify the diagnostic value of NAFLD molecular prediction model FLI, NAFLD LFS and Liver fat (\%) in Chinese Han population that had been reported previously $[17,18]$. We found that these NAFLD prediction models possess the relatively good diagnostic sensitivity and specificity for the risk of NAFLD. Meanwhile, these molecular prediction models could be optimized for the optimal diagnostic accuracy.

BMI is a tightly correlative factor with the risk of NAFLD and could be regarded as the indicator for the development of NAFLD [33, 34]. In our study, we divided all the participants into NAFLD group and health group with according to the MRI-PDFF examination results. The average BMI value in NAFLD group was significantly higher than health group, which is accord with the classical conclusion. In addition, the prevalence of T2D and MS in NAFLD group was also higher than in health group, which suggested that NAFLD patients possess the high risk of T2D and MS.

Bedogni et al. reported the fatty liver index which was a predictor of hepatic steatosis in the general population. According to the model, a FLI $<30$ could be rule out hepatic steatosis with the sensitivity was $87 \%$ and a FLI $\geq 60$ could be rule in hepatic steatosis with the specificity $86 \%$ [17]. In our study, we used this model to predict the risk of NAFLD in the MRI-PDFF verified population, and we found that the optimal cut-off value was FLI $\geq 37.64$ with a sensitivity of $87.0 \%$ and specificity of $58.5 \%$. In addition, the FLI score 60 possesses a sensitivity of $59.8 \%$ and specificity of $81.1 \%$. in our MRI-PDFF diagnosed population. The differences of the diagnostic sensitivity and specificity of FLI model verified by Bedogni et al. and us may contributed by the diagnostic criterion and methods, and the differences of ethnic and region, which may result in some biases in the NAFLD diagnosis. These results suggested that the accuracy of molecular prediction model could be improved when MRI-PDFF was selected as the NAFLD diagnostic golden standard. Kotronen et al. developed a NAFLD liver fat score (NAFLD LFS) model to screen NAFLD patients from general population with the available clinical and laboratory parameters. They found that the NAFLD could be confirmed with the sensitivity of $86 \%$ and specificity of $71 \%$ when the cutoff value was greater than -0.640 [18]. In this study, 
we predicted the risk of NAFLD using the NAFLD LFS model in the MRI-PDFF verified population, and the optimal cut-off value for NAFLD diagnosis was -1.025 with the sensitivity of $78.7 \%$ and specificity of $73.3 \%$. When sensitivity was the same as that of the original text $(86 \%)$, the cutoff value was -1.4342 , which was lower than the optimal cutoff value. Although sensitivity was increased and cut-off value decreased, specificity was decreased. The diagnostic sensitivity and specificity was comparative, and the smaller differences may also result in by the diagnostic criteria and methods, and the differences of ethnic and region, which may lead to the misdiagnosis of NAFLD, and therefore the accuracy of the score was interfered. In addition to this, Hernando et al. found that although it could be corrected, the small PDFF biases observed across vendors [35].

In summary, we used MRI-PDFF as the NAFLD diagnostic gold standard to verify the diagnostic values of molecular prediction model in NAFLD patients. Our results suggested that the FLI, NAFLD LFS, and Liver fat (\%) models were comparative accurate and could be regard as the useful tool to scan the NAFLD patients from general population in Chinese Han population. Especially, the accuracy of molecular prediction models could be improved when the MRI-PDFF was used as the diagnostic golden standard. This study reevaluated the three existing models and provided a more reliable truncation value for the Chinese population. This might help to assess the risk of NAFLD through routine physical examinations, saving medical resources and reducing the burden on patients. In future, more molecule prediction models which based on easily available clinical and laboratory data should be constructed according to accurate diagnosis of NAFLD by MRI-PDFF.

\section{Acknowledgements}

Not applicable.

\section{Authors' contributions}

$K L$ and $Y X$ carried out the design of this study and the critical revision of the manuscript. QZ and YZ were responsible for collecting data and writing papers. WY and ZX were responsible for testing MRI-PDFF and the other indicators. ZZ was responsible for data collation and statistical analysis. SL was responsible for recruiting volunteers and coordinating the department's operations. All authors read and approved the final manuscript.

\section{Funding}

This study was supported by Grants of National Natural Science Foundation of China (31770837).

\section{Availability of data and materials}

The datasets used and/or analysed during the current study are available from the corresponding author on reasonable request.

\section{Ethics approval and consent to participate}

This study was approved by the Ethics Committee of Qingdao municipal hospital and the written informed consents were obtained from all Participants.

\section{Consent for publication}

All participants have written informed consent for their personal and clinical details to be published in this study.

\section{Competing interests}

All authors have no conflicts of interest.

\section{Author details}

${ }^{1}$ Department of Radiology, Qingdao Municipal Hospital, 5 Donghaizhong Road, Qingdao 266071, Shandong Province, China. ${ }^{2}$ Department of Infectious Disease, Qingdao Municipal Hospital, 1 Jiaozhou Road, Qingdao 266011, Shandong Province, China. ${ }^{3}$ Clinical Research Center, Qingdao Municipal Hospital, 5 Donghaizhong Road, Qingdao 266071, Shandong Province, China.

Received: 28 March 2020 Accepted: 17 February 2021

Published online: 25 February 2021

\section{References}

1. Younossi Z, Tacke F, Arrese M, Chander Sharma B, Mostafa I, Bugianesi E, Wai-Sun Wong V, Yilmaz Y, George J, Fan J, et al. Global perspectives on nonalcoholic fatty liver disease and nonalcoholic steatohepatitis. Hepatology. 2019;69(6):2672-82.

2. Younossi Z, Anstee QM, Marietti M, Hardy T, Henry L, Eslam M, George J, Bugianesi E. Global burden of NAFLD and NASH: trends, predictions, risk factors and prevention. Nat Rev Gastroenterol Hepatol. 2018;15(1):11-20.

3. Fiorucci S, Biagioli M, Distrutti E. Future trends in the treatment of nonalcoholic steatohepatitis. Pharmacol Res. 2018;134:289-98.

4. Friedman SL, Neuschwander-Tetri BA, Rinella M, Sanyal AJ. Mechanisms of NAFLD development and therapeutic strategies. Nat Med. 2018;24(7):908-22.

5. Rinella ME. Nonalcoholic fatty liver disease: a systematic review. JAMA. 2015;313(22):2263-73.

6. Merriman RB, Ferrell LD, Patti MG, Weston SR, Pabst MS, Aouizerat BE, Bass NM. Correlation of paired liver biopsies in morbidly obese patients with suspected nonalcoholic fatty liver disease. Hepatology. 2006;44(4):874-80.

7. Bedossa P. Diagnosis of non-alcoholic fatty liver disease/non-alcoholic steatohepatitis: why liver biopsy is essential. Liver Int. 2018;38(Suppl 1):64-6.

8. Bonekamp S, Tang A, Mashhood A, Wolfson T, Changchien C, Middleton MS, Clark L, Gamst A, Loomba R, Sirlin CB. Spatial distribution of MRIdetermined hepatic proton density fat fraction in adults with nonalcoholic fatty liver disease. J Magn Reson Imaging. 2014;39(6):1525-32.

9. Alisi A, Pinzani M, Nobili V. Diagnostic power of fibroscan in predicting liver fibrosis in nonalcoholic fatty liver disease. Hepatology 2009, 50(6):2048-2049. author reply 2049-2050.

10. Chan WK, Nik Mustapha NR, Mahadeva S. Controlled attenuation parameter for the detection and quantification of hepatic steatosis in nonalcoholic fatty liver disease. J Gastroenterol Hepatol. 2014;29(7):1470-6.

11. Caussy C, Reeder SB, Sirlin CB, Loomba R. Noninvasive, quantitative assessment of liver fat by MRI-PDFF as an endpoint in NASH trials. Hepatology. 2018;68(2):763-72

12. Noureddin M, Lam J, Peterson MR, Middleton M, Hamilton G, Le TA, Bettencourt R, Changchien C, Brenner DA, Sirlin C, et al. Utility of magnetic resonance imaging versus histology for quantifying changes in liver fat in nonalcoholic fatty liver disease trials. Hepatology. 2013;58(6):1930-40.

13. Yokoo T, Shiehmorteza M, Hamilton G, Wolfson T, Schroeder ME, Middleton MS, Bydder M, Gamst AC, Kono Y, Kuo A et al. Estimation of hepatic proton-density fat fraction by using MR imaging at 3.0 T. Radiology 2011, 258(3):749-759.

14. Imajo K, Kessoku T, Honda Y, Tomeno W, Ogawa Y, Mawatari H, Fujita K, Yoneda M, Taguri M, Hyogo $\mathrm{H}$ et al. Magnetic resonance imaging more accurately classifies steatosis and fibrosis in patients with nonalcoholic fatty liver disease than transient elastography. Gastroenterology 2016, 150(3):626-637.

15. Middleton MS, Van Natta ML, Heba ER, Alazraki A, Trout AT, Masand P, Brunt EM, Kleiner DE, Doo E, Tonascia J, et al. Diagnostic accuracy of 
magnetic resonance imaging hepatic proton density fat fraction in pediatric nonalcoholic fatty liver disease. Hepatology. 2018;67(3):858-72.

16. Gu J, Liu S, Du S, Zhang Q, Xiao J, Dong Q, Xin Y. Diagnostic value of MRI-PDFF for hepatic steatosis in patients with non-alcoholic fatty liver disease: a meta-analysis. Eur Radiol. 2019;29(7):3564-73.

17. Bedogni G, Bellentani S, Miglioli L, Masutti F, Passalacqua M, Castiglione A, Tiribelli C. The Fatty Liver Index: a simple and accurate predictor of hepatic steatosis in the general population. BMC Gastroenterol. 2006;6:33.

18. Kotronen A, Peltonen M, Hakkarainen A, Sevastianova K, Bergholm R, Johansson LM, Lundbom N, Rissanen A, Ridderstrale M, Groop L, et al. Prediction of non-alcoholic fatty liver disease and liver fat using metabolic and genetic factors. Gastroenterology. 2009;137(3):865-72.

19. Niu L, Geyer PE, Wewer Albrechtsen NJ, Gluud LL, Santos A, Doll S, Treit PV, Holst JJ, Knop FK, Vilsboll T, et al. Plasma proteome profiling discovers novel proteins associated with non-alcoholic fatty liver disease. Molecular systems biology. 2019;15(3):e8793.

20. van den Berg EH, Flores-Guerrero JL, Gruppen EG, de Borst MH, WolakDinsmore J, Connelly MA, Bakker SJL, Dullaart RPF: Non-Alcoholic Fatty Liver Disease and Risk of Incident Type 2 Diabetes: Role of Circulating Branched-Chain Amino Acids. Nutrients 2019, 11(3).

21. El Kamari V, Hileman CO, Gholam PM, Kulkarni M, Funderburg N, McComsey GA. Statin therapy does not reduce liver fat scores in patients receiving antiretroviral therapy for HIV infection. Clin Gastroenterol Hepatol. 2019, 17(3):536-542.

22. Ma Y, Belyaeva OV, Brown PM, Fujita K, Valles K, Karki S, de Boer YS, Koh C, Chen Y, Du X, et al. 17-Beta hydroxysteroid dehydrogenase 13 is a hepatic retinol dehydrogenase associated with histological features of nonalcoholic fatty liver disease. Hepatology. 2019;69(4):1504-19.

23. Rickham PP: Human experimentation. Code of Ethics of the World Medical Association. Declaration of Helsinki. Br Med J. 1964; 2(5402):177.

24. Chavez S, Stanisz G: Comparing average breast fat content results from two different protocols at 1.5T and 3T: can the data be pooled? J Magn Reson Imaging. 2014, 40(4):890-898.

25. Tang A, Desai A, Hamilton G, Wolfson T, Gamst A, Lam J, Clark L, Hooker J, Chavez T, Ang BD, et al. Accuracy of MR imaging-estimated proton density fat fraction for classification of dichotomized histologic steatosis grades in nonalcoholic fatty liver disease. Radiology. 2015;274(2):416-25.

26. Jia W, Weng J, Zhu D, Ji L, Lu J, Zhou Z, Zou D, Guo L, Ji Q, Chen L et al. Standards of medical care for type 2 diabetes in China 2019. Diabetes Metab Res Rev 2019:e3158.
27. Nobili V, Alkhouri N, Alisi A, Della Corte C, Fitzpatrick E, Raponi M, Dhawan A. Nonalcoholic fatty liver disease: a challenge for pediatricians. JAMA Pediatr. 2015;169(2):170-6.

28. Tarantino G, Finelli C. Pathogenesis of hepatic steatosis: the link between hypercortisolism and non-alcoholic fatty liver disease. World J Gastroenterol. 2013;19(40):6735-43.

29. Middleton MS, Heba ER, Hooker CA, Bashir MR, Fowler KJ, Sandrasegaran K, Brunt EM, Kleiner DE, Doo E, Van Natta ML, et al. Agreement between magnetic resonance imaging proton density fat fraction measurements and pathologist-assigned steatosis grades of liver biopsies from adults with nonalcoholic steatohepatitis. Gastroenterology. 2017;153(3):753-61.

30. Kantartzis K, Rettig I, Staiger H, Machann J, Schick F, Scheja L, Gastaldelli A, Bugianesi E, Peter A, Schulze MB, et al. An extended fatty liver index to predict non-alcoholic fatty liver disease. Diabetes Metab. 2017:43(3):229-39.

31. Vilar-Gomez E, Chalasani N. Non-invasive assessment of non-alcoholic fatty liver disease: Clinical prediction rules and blood-based biomarkers. J Hepatol. 2018:68(2):305-15.

32. Saokaew S, Kanchanasuwan S, Apisarnthanarak P, Charoensak A, Charatcharoenwitthaya P, Phisal prapa P, Chaiyakunapruk N. Clinical risk scoring for predicting non-alcoholic fatty liver disease in metabolic syndrome patients (NAFLD-MS score). Liver Int. 2017;37(10):1535-43.

33. Schetz M, De Jong A, Deane AM, Druml W, Hemelaar P, Pelosi P, Pickkers P, Reintam-Blaser A, Roberts J, Sakr Y, et al. Obesity in the critically ill: a narrative review. Intensive Care Med. 2019;45(6):757-69.

34. Croci I, Byrne NM, Choquette S, Hills AP, Chachay VS, Clouston AD, O'Moore-Sullivan TM, Macdonald GA, Prins JB, Hickman IJ. Whole-body substrate metabolism is associated with disease severity in patients with non-alcoholic fatty liver disease. Gut. 2013;62(11):1625-33.

35. Hernando D, Sharma SD, Aliyari Ghasabeh M, Alvis BD, Arora SS, Hamilton G, Pan L, Shaffer JM, Sofue K, Szeverenyi NM et al. Multisite, multivendor validation of the accuracy and reproducibility of proton-density fatfraction quantification at $1.5 \mathrm{~T}$ and $3 \mathrm{~T}$ using a fat-water phantom. Magn Reson Med. 2017; 77(4):1516-24.

\section{Publisher's Note}

Springer Nature remains neutral with regard to jurisdictional claims in published maps and institutional affiliations.
Ready to submit your research? Choose BMC and benefit from:

- fast, convenient online submission

- thorough peer review by experienced researchers in your field

- rapid publication on acceptance

- support for research data, including large and complex data types

- gold Open Access which fosters wider collaboration and increased citations

- maximum visibility for your research: over $100 \mathrm{M}$ website views per year

At BMC, research is always in progress.

Learn more biomedcentral.com/submissions 